\title{
MORTALIDAD PERINATAL - ASPECTOS GENERALES
}

\author{
Dr. Arturo Rodríguez Soto* \\ Dr. Luis Armando Muñoz González**
}

\section{Definición :}

El concepto de Mortalidad Perinatal se ha modificado en el curso de los años; Nesbitt (10) en 1956 designaba con este término las muertes de fetos y recién nacidos de un peso igual o superior a 1.000 gramos ocurridas desde la semana 28 , hasta los 28 días de vida extrauterina.

En 1961, Schelesinger y Nesbitt (22) incluyen en la definición las muertes desde la semana 20 de gestación hasta el día 28 de vida extrauterina.

Shapiro, Schelesinger y Nesbitt (19) en 1958 presentan dos definiciones: Mortalidad Fetal I que contempla las muertes fetales de 28 semanas o más de gestación y los niños muertos hasta el séptimo día de vida y mortalidad perinatal II que incluye las muertes fetales de 20 semanas o más y los niños muertos hasta el día 28 de vida.

La Federación Internacional de Sociedades de Obstetricia y Ginecología reunida en Moscú en Agosto de 1973, recomienda que se defina como período perinatal el que comienza cuando el feto pesa 1.000 gramos (equivalente a 28 semanas de gestación) y termina cuando el recién nacido ha completado 7 días de vida (168 horas). (3).
En ausencia del dato de peso, se considera que 35 ctms. de longitud del feto equivalen a 1.000 gramos.

En ausencia de estos dos datos se considera que un feto ha entrado en el período perinatal, cuando la edad gestacional alcanza 28 semanas completas (196 días).

Las muertes ocurridas durante el período perinatal antes o después del nacimiento se incluyen dentro de las tasas de mortalidad perinatal básica.

Las definiciones propuestas por la FIGO son las que hemos acogido para el presente trabajo, el cual hace parte del Relato Oficial de la Sociedad Colombiana de Obstetricia y Ginecología para el XI Congreso Co lombiano de Obstetricia y Ginecología.

\section{Factores principales relacionados con la Mortalidad Perinatal}

En el período perinatal se describen como causas básicas de Mortalidad Perinatal las siguientes: (Cuadro No. 1 ).

* Profesor Asociado del Departamento de Ginecología y Obstetricia, Facultad de Medicina de la Universidad Nacional.

** Profesor Asistente del Departamento de Ginecología y Obstetricia, Facultad de Medicina de la Universidad Nacional. 
CUADRO NN 1

MORTALIDAD PERINATAL

\begin{tabular}{|c|c|}
\hline \multirow[b]{2}{*}{ BIOMEDICOS } & \multirow[b]{2}{*}{ BIOSOCIALES } \\
\hline & \\
\hline * Anomalías congénitas & * Edad materna \\
\hline * Peso bajo al nacer & * Orden de nacimiento \\
\hline $\begin{array}{l}\text { * Afecciones agregadas } \\
\text { al embarazo }\end{array}$ & * Edad gestacional \\
\hline $\begin{array}{l}\text { * Afecciones maternas } \\
\text { relacionadas con embarazo }\end{array}$ & * Estado socioeconómico \\
\hline * Patología del embarazo & * Area geográfica \\
\hline * Patología del parto & * Asistencia prenatal \\
\hline * Patología neonatal & * Antecedentes obstétricos \\
\hline
\end{tabular}

¡̊ Anomalías congénitas. Cabe distinguir las malformaciones causadas por la transmisión de informaciones genéticas anormales o por aberraciones cromosómicas y por otra parte las causadas por fenómenos ambientales, solos o combinados con factores predisponentes como la edad de los progenitores, la multiparidad, infecciones virales, drogas, irradiación, etc.

Hasta hace poco tiempo se subestimó considerablemente la magnitud de este problema y las estadísticas no daban información sino de las anomailías severas reconocidas en el momento del nacimiento (28).

En Estados Unidos con la publicación del trabajo de Mc Intosch (9) en 1954 se determinó que el problema era de proporciones mayores, ya que por cada 100 niños se diagnosticaron 8 anomalías congénitas definitivas. En un estudio similar, Schapiro (20) encontró anomalías en 7 de cada 100 niños.

En un estudio de Yunis y Giraldo (30) efectuado sobre 25.763 nacimientos en el Instituto Materno Infantil de Bogotá, se encontraron 1.115 niños con anomalías congénitas $4,3 \%$, de las cuales 2,2\% eran anomalías mayores y 2,1\% anomalías menores.

Al efectuar las correcciones correspondientes se concluye que 8 a 9 de cada 100 nacidos vivos padecen durante su vida algún tipo de anomalía congénita. Habrá un número también importante de niños con anomalías congénitas incompatibles con la vida y que morirán in útero o en los primeros días de vida extrauterina.

Ciertas anomalías congénitas están asociadas positivamente a la edad de los padres; el hallazgo más importante es el que relaciona los síndromes de Down y Klinefelter con la mayor edad de la madre. Un descenso en la frecuencia de nacimiento en mujeres de edad avanzada podrá conducir a una reducción significativa en la incidencia de estos síndromes (14).

2 . Peso insuficiente al nacer. Durante muchos años el parámetro primordial de tipo estadístico para clasificar el recién nacido con relación a la madurez ha sido el peso. La imperfección de este parámetro se reconoce universalmente, ya que clínicamente se ha comprobado que pesos específicos revelan diferentes grados de madurez, según los subgrupos de po- 
blación y según las características nutricionales.

Sin embargo, la facilidad de obtención del peso en grandes grupos de población, las menores posibilidades de error que con otros parámetros y la elevada mortalidad perinatal en ciertos grupos específicos de peso, hacen que se haya conservado este parámetro sin dejar de reconocer sus limitaciones $(17,21)$.

Es importante hacer la distinción en tres categorías de los niños de peso insuficiente.

a) El nacido antes de tiempo

b) El congénitamente pequeño

c) El de peso bajo para la edad de gestación

Hay que tener en cuenta que los factores asociados con el nacimiento de niños de peso insuficiente no son solamente congénitos ni nutricionales, sino que hay otros factores como el peso, la talla, la edad y la paridad de la madre (23).

3: Mortalidad Perinatal por afecciones maternas relacionadas con el embarazo. En este grupo se destacan la toxemia gravídica y las infecciones maternas ante-parto e intra-parto, tales como pielitis, pielonefritis, corioamniotitis.

4\% Mortalidad Perinatal por afecciones maternas agregadas a la gestación. $\mathrm{Se}$ incluyen en este grupo las enfermedades cardiovasculares y renales crónicas, la diabetes, sífilis, rubeola, toxoplasmosis, listeriosis, etc.

5: Patología del embarazo. Comprende las complicaciones del embarazo múltiple, del hidramnios, la ruptura prematura de membranas, la placenta previa, el abruptio, las afecciones del cordón, que son causantes por sí o por las manifestaciones de anoxia e hipoxia que producen, de elevada mortalidad perinatal.

6: Mortalidad Perinatal por afecciones que ocasionan anoxia e hipoxia. Los estudios clínicos y patológicos indican que la hipoxia es una de las mayores causas de mortalidad perinatal. El número de complicaciones en el embarazo que ocasionan anoxia intrauterina incluyen:

a) Reducción del oxígeno en la circulación materna, como ocurre en la anemia severa, en el shock, la hemorragia, enfermedades pulmonares 0 anestesia inadecuada.

b) Reducción del oxígeno en la circulación fetal como consecuencia de falla de la función placentaria: abruptio placenta previa, infartos placentarios, toxemia, diabetes.

c) Reducción del oxígeno fetal como consecuencia de anomalías del cordón: Procidencia, nudos, circulares.

d) Reducción del oxígeno fetal como consecuencia de anemia fetal, enfermedad hemolítica, ruptura de vasos fetales, vasa previa. El pronóstico fetal depende en gran parte de la severidad del proceso y de la edad de gestación (12).

7ํ. Hemorragia intracraneana. Se presenta esta complicación generalmente en cualquier condición que interfiera con el transporte de oxígeno al feto, en los traumatismos fetales o en las complicaciones maternas severas. Estos fetos a menudo presentan extensas hemorragias intracraneales aún sin evidencia de traumatismo mecánico $(6,24)$.

\section{8? Patología Neonatal.}

La eritroblastosis fetal, trastorno común del feto y del recién nacido es 
la consecuencia de la isoinmunización materna a los antígenos del factor $\mathrm{RH}$, del sistema $\mathrm{ABO} O$ en casos raros a los demás factores de diferenciación de los grupos sanguíneos y se constituye en una causa significativa de mortalidad perinatal, no obstante que se hayan hecho avances importantes en su manejo y en su prevención tales como la transfusión in útero y la aplicación de gamaglobulina anti D. $(4,5,29,31)$.

La asfixia neenatal que es la adaptación incompleta de la respiración a las condiciones extrauterinas por falta de reacción de los centros respiratorios a los estímulos exteriores o internos, o por anomalías pulmonares que impiden la aireación eficaz de los alvéolos y el establecimiento de la perfusión pulmonar.

El síndrome de dificultad respiratoria en todos sus grados tales como: dificultad respiratoria secundaria, dificultad respiratoria por aspiración, por membrana hialina, por inmadurez, etc. (3).

La infección que se constituye en algunos sitios en una causa muy relievante de mortalidad perinatal. El recién nacido pasa generalmente de un medio estéril al medio extrauterino en el que abundan los saprófitos y otros agentes infecciosos que muy rápidämente colonizan el organismo por etapas sucesivas, empezando por la nasofaringe, siguiendo por la región umbilical y terminando por la superficie cutánea. Entre la colonización normal y el desencadenamiento de una infección post-natal que se puede propagar a toda una sala de lactantes no hay más que diferencias de grado (1).

\section{Factcres biosociales y Mortalidad Perinatal}

Existe evidente relación entre la mortalidad perinatal y algunos facto- res biosociales tales como: la edad de la madre, la paridad, el estado socio-económico, el área geográfica, la raza, la asistencia prenatal etc.

Las mujeres menores de 20 años y las mayores de 35 , se constituyen en un grupo de riesgo elevado, con factores asociados importantes, como toxemia gravídica, enfermedad crónica vascular hipertensiva, prematurez, etc. $(18,24,25)$.

En cuanto a la paridad todos los autores anotan una evidente relación entre las tasas de mortalidad y el orden del nacimiento; se advierten tasas muy elevadas de mortalidad perinatal después del 5 : nacimiento $(2,4$, $8,16,27$ ).

\section{Morbilidad Perinatal}

El análisis de los factores relacionados con el nacimiento del niño defectuoso es complejo. Las dimensiones del problema solo se pueden apreciar por estudios longitudinales con seguimiento de un número grande de niños. Algunos defectos son obvios en el recién nacido; otros solo se manifiestan a medida que el niño se relaciona con el ambiente y entonces aparecen trastornos de índole psicomotora, neurológica o intelectual (7, $15)$.

En el proyecto perinatal de Estados Unidos (26) que comprenden el seguimiento de 60.000 gestaciones, mostró en su primer año que 1,5\% de los niños eran neurológicamente anormales y que en el $8,1 \%$ había trastornos motores.

La magnitud del problema de la morbilidad perinatal se ilustra por cálculos hechos sobre el número de niños impedidos en Estados Unidos para 1970: 2'642.000 retardados mentales, 347.000 con parálisis cerebral y 428.000 con epilepsia. 
Entre las observaciones importantes que se han hecho figuran las siguientes: en niños prematuros hay aumento importante de anomalías neurológicas $(30 \%$ de los niños de un año de edad con problemas neurológicos nacieron prematuramente). Las mujeres de más de 40 años tienen grandes probabilidades de concebir niños con anomalías congénitas. La anoxia y la asfixia tienen importancia esencial en el daño cerebral. Los niños nacidos en presentación de pelvis, los nacidos con cesárea o los que presentan placenta previa constituyen un grupo muy vulnerable respecto a morbilidad y mortalidad perinatal (26).

\section{Aspectos internacionales} de la Mortalidad Perinatal

La amplitud del cuadro internacional de mortalidad perinatal se des- cribe adecuadamente al definir los dos extremos de la gama. En un extremo están los países con condiciones sanitarias avanzadas y en el otro extremo están los países en desarrollo con mortalidad perinatal elevada.

Las tasas de mortalidad perinatal oscilan entre 18.9 y 43.6 por 1.000 nácidos vivos en los países europeos. En el Cuadro № 2 se transcriben los datos de mortalidad perinatal en algunos países europeos. Se aprecia que las cifras de mortalidad perinatal con sistemas de registro fidedigno y totalmente confiables en países como Suecia, Bulgaria, Finlandia, Noruega, Checoeslovaquia son inferiores a $25 \mathrm{x}$ 1.000. Países como Italia, Portugal, y posiblemente España continúan presentando tasas por encima de $35 \times$ 1.000 ( 11 ).

\section{CUADRO № 2}

MORTALIDAD PERINATAL

\begin{tabular}{lccc}
\hline País & $\begin{array}{l}\text { Fetal } \\
\text { tardía }\end{array}$ & $\begin{array}{l}\text { Neonatal } \\
\text { temprana }\end{array}$ & Perinatal \\
\hline SUECIA & 10.2 & 8.5 & 18.7 \\
BULGARIA & 9.9 & 9.1 & 19.0 \\
FINLANDIA & 10.3 & 10.5 & 20.8 \\
NORUEGA & 12.1 & 9.0 & 21.1 \\
ALEMANIA & 12.7 & 12.8 & 25.5 \\
FRANCIA & 15.2 & 12.5 & 27.7 \\
IRLANDA & 16.0 & 13.1 & 29.1 \\
GRECIA & 15.9 & 14.5 & 30.4 \\
ITALIA & 19.7 & 17.1 & 36.8 \\
PORTUGAL & 27.3 & 16.3 & 43.6 \\
\hline
\end{tabular}

\section{Mortalidad Perinatal en América}

Cuadro № 3. El estudio de Mortalidad perinatal en América, ofrece dos facetas diferentes: países como Canadá y Estados Unidos con registros confiables y con tasas de 25.6 y 26.5. $Y$ el resto de países que con excepción de Chile y Cuba tienen esta- dísticas poco confiables y fidedignas y con tasas de mortalidad que oscilan entre 35 y 50 × 1.000 (11).

\section{Mortalidad Perinatal en Colombia}

Cuadro № 4. La última tasa de mortalidad perinatal informada en Colombia es de 38.6 × 1.000 y co- 


\section{CUADRO № 3}

MORTALIDAD PERINATAL

\begin{tabular}{lccc}
\multicolumn{4}{c}{ ANERICA } \\
\hline País & $\begin{array}{l}\text { Fetal } \\
\text { tardía }\end{array}$ & $\begin{array}{l}\text { Neonatal } \\
\text { temprana }\end{array}$ & Perinatal \\
\hline CANADA & 11.3 & 14.3 & 25.6 \\
ESTADOS UNIDOS & 10.6 & 15.5 & 26.5 \\
MEXICO & 17.0 & 14.4 & 32.5 \\
CUBA & 21.1 & 13.5 & 34.6 \\
COSTA RICA & 19.8 & 16.6 & 36.6 \\
ARGENTINA & 23.7 & 14.5 & 38.2 \\
COLOMBIA & 17.2 & 20.9 & 38.6 \\
ECUADOR & 23.8 & 16.5 & 40.3 \\
PUERTO RICO & 25.3 & 17.5 & 42.8 \\
CHILE & 24.2 & 24.1 & 48.3 \\
GUATEMALA & 30.8 & 20.0 & 50.7 \\
\hline
\end{tabular}

rresponde al informe demográfico de las Naciones Unidas. Se lograron obtener del Dane las tasas de algunos
Departamentos en 1972 y las cuales oscilan entre 39.7 y 50.7 con un promedio de 44.9 .

\section{CUADRO N: 4}

MORTALIDAD PERINATAL

COLOMBIA

\begin{tabular}{lccc}
\hline & $\begin{array}{c}1966 \\
\text { Fetal } \\
\text { tardía }\end{array}$ & $\begin{array}{l}\text { Neonatal } \\
\text { temprana }\end{array}$ & Perinatal \\
\hline COLOMBIA & 17.7 & 20.9 & 38.6 \\
& 1972 & & \\
& Fetal & Neonatal & \\
tardía & temprana & Perinatal \\
ANTIOQUIA & 18.35 & 21.42 & 39.77 \\
VALLE & 23.82 & 19.38 & 43.20 \\
SANTANDER & 25.97 & 24.80 & 50.77 \\
CALDAS & 25.56 & 20.55 & 46.11 \\
\hline
\end{tabular}

Estas cifras de pérdidas perinatales similar en el bloque de Naciones llamadas del Tercer Mundo han hecho que los obstetras, pediatras y especialistas en Medicina Preventiva estudien intensamente el problema. Hoy ha surgido como especialidad vital la perinatología, la cual ha establecido un verdadero puente entre la Obste- tricia y la Pediatría y hace presumir que con sus adelantos se logre un mejor pronóstico para este grupo humano que se pierde o se lesiona a partir de la semana 28 de gestación y la primera semana neonatal.

Los nuevos recursos de la medicina perinatal (Cuadro № 5) se pueden 
resumir en identificación del embarazo de alto riesgo, valoración del medio ambiente fetal y del feto in útero; el estudio de los índices bioquímicos, endocrinos y enzimáticos, las técnicas del ultrasonido, la monitoría fetal, el diagnóstico prenatal de ano- malías genéticas y la farmacología neonatal y fetal con la posibilidad de tratamiento intrauterino de enfermedades fetales y de tratamiento del neonato de acuerdo a su capacidad de metabolizar las drogas.

\section{CUADRO NN 5}

\section{NUEVOS RECURSOS EN PERINATOLOGIA}

\begin{tabular}{ll}
\hline EMbarazo DE ALTO RIESGO & $\begin{array}{c}\text { VALORACION DEL } \\
\text { MEDIO AMBIENTE FETAL }\end{array}$ \\
* clasificación & $\begin{array}{l}\text { * índices enzimáticos, bioquímicos } \\
\text { indices hormonales } \\
\text { * calificación } \\
\text { VALORACION DEL ESTADO } \\
\text { DEL FETO IN UTERO } \\
\text { * líquido amniótico }\end{array}$ \\
* ultrasonido & FARMACOLOGIA \\
* monitoría & FETAL Y NEONATAL
\end{tabular}

\section{BIBLIOGRAFIA}

1 BENIRSCHKE, K.: Routes and types of infection in the fetus and new born. Am. J. Dis. Children. 99: 714, 1960.

2 BRICEÑO, R. T., RIVAS, H., y GRIECO, M. Mortalidad perinatal. Análisis de 1.148 casos. Rev. de Obst. y Ginec. de Venezuela. 32: 193, 1972.

3 F.I.G.O. News definition of terms in human reproduction. International Journal of Gynecology \& Obstetrics. 12: 30, 1974.

4 FREDA, V. J., GORMAN, J. G., and POLLACK. W.: Successful prevention of experimental $\mathrm{RH}$ sensitization in man with and Anti-RH. Gama 2 globulin antibody preparation. Transfusion. 4: 26, 1964.

5 FREDA, V. J., GORMAN, J. G., and POLLACK, W.: RH Factor: Prevention of isoinmunization and clinical trial on mothers. Science. 151: 828, 1966.

6 HALLER, E. S., NESBITT, R. E. L., and ANDERSON. G. W.: Clinical and Pathologic concepts of Gross intracranial hemorrhage in perinatal Mortality. Obst. and Gyn. Survey. $11: 179,1956$.

7 LILIENFELD, A. M., and PARKHURST, E. : A study of the association of factors of preg- nancy and parturition with the development of cerebral palsy. Am. J. Hyg. 53: 262, 1951.

8 MAZZOICO DE DUSSANT, N.: Mortalidad perinatal. Obstetricia y Ginecología Latinoamericanas. 31: 269, 1973.

9 MC INTOSH, R., MERRITT, K. K.. RICHARDS, M. R., SAMUELS, M. H., and BELLOWS, M. R.: Incidence of congenital malformations. A study of 5.964 pregnancies. Pediatrics. $14: 505,1954$

10 NESBITT, R. E. L., and ANDERSEN. G. W.: Perinatal Mortality. Clinical and Pathologic aspects. Obst. and Gynec. 5: 50, 1956.

11 Organización Mundial de la Salud. La prevención de la morbilidad y de la mortalidad perinatales. Informe técnico Nọ 42. Ginebra 1972.

12 Organización Mundial de la Salud. Efectos del parto en el feto y el recién nacido. Informe técnico No 300. Ginebra, 1965.

13 Organización Mundial de la Salud. Prevención de la mortalidad y morbilidad perinatales. Informe técnico No 457. Ginebra, 1970.

14 Organización Mundial de la Salud. Desórdenes Genéticos. Prevención, tratamiento y 
rehabilitación. Informe técnico No 497. Ginebra 1972.

15 PASAMANICK, B., and LILIENFELD, A. M. : Association of maternal and fetal factors with development of mental deficiency. J.A.M.A. 159: 155, 1955.

16 PUFFER, R., y SERRANO, C.. V.: Características de la mortalidad perinatal en la niñez. OPS. Pubilcación científica 262, Washington, D.C. 1973.

17 ROSA, F., and RESNICK, L.: Birth weight and perinatal Mortality in the American Indian. Am. J. Obs. \& Gyn. 55: 972, 1965.

18 ROSS, R. A.: Some important factors in perinatal mortality statics in a rural state. Amer. J. Obstet. Gynec. 88: 342, 1964.

19 SHAPIRO, S., SCHELESINGER, E. R., and NESBITT, R. E. L.: Infant, perinatal, maternal and childhood mortality in the United States. Vital and Health Statistics Monographs. American Public Health Association. 1968. Harvard University Press. Cambridge Massachusetts.

20 SHAPIRO, S., ROSS, L., and LEVINE, H. S. Relation Ship of selected prenatal factors to pregnancy autcome and congenital anomalies. Am. J. Publ. Health. 55: 268, 1965.

21 SCHELESINGER, E. R., and ALLAWAY, N. C.: The combined effect of birth weight and length of gestation on neonatal mortality among single premature births. Pediatrics. 15: 698, 1955.

22. SCHELESINGER, E. R., and NESBITT, R. E. L.: Perinatal mortality. Clinical Obst. and Gynec. 4: 318, 1961.
23 SCHWARTZ, S.: Prenatal care, prematurity and neonatal mortality. Am. J. Obst. \& Gynec. 83: 591, 1962.

24 STEER, C. M. and MOORE, J. G.: Course of perinatal mortality: a review of etiologic factors in the Sloane Hospital 1.888. 1967. Obst. and Gynec. 34: 113, 1969.

25 STINE, O. C., RIDER, R. V. and SWEENEY, E.: School leaving due to pregnancy in a urban adolescent population. Am. J. Publ. Health. 54: 1, 1964.

26 U. S. Childrens Bureau Infant Mortality. A challenge to the nation. Washington, D. C. 1966.

27 VASQUEZ, J., OVIES, A., GALINDO, C., CANDEBAT, Z., y HEREDIA, M.: Informe preliminar del estudio de la mortalidad perinatal en la provincia de La Habana. Rev. Cub. Ped. 44 : 53, 1972.

28 WARKANY, J., and KALTER, H.: Congenital malformations. New England J. Med. 265: 993, 1961.

29 WOODRCW, J. C., and DONOHOE, W. X. I. A.: RH immunization by pregnancy. Results a survey their relevance to profilactic therapy. Brit. Med. J. 4: 139, 1968.

30 YUNIS y GIRALDO. Comunicación personal. 1975.

31 ZIPUSKY, A., and ISRAELS, L. G.: The pathogenesis and prevention of $\mathrm{RH}$ inmunization. Canad. Med. Assoc. J. 97 : 1.245, 1967. 